\title{
Analysis of the Efficacy and Renal Prognosis of a Reduced-Dose Immunosuppressive Regimen of Mild to Moderate ANCA-Associated Renal Vasculitis
}

\section{Ziyang Li}

Shanghai Jiaotong University School of Medicine Affiliated Renji Hospital

\section{Jiwen Bao}

Shanghai Jiaotong University School of Medicine Affiliated Renji Hospital

Huanzhen Yao

Shanghai Jiaotong University School of Medicine Affiliated Renji Hospital

\section{Minfang Zhang}

Shanghai Jiaotong University School of Medicine Affiliated Renji Hospital

\section{Leyi Gu}

Shanghai Jiaotong University School of Medicine Affiliated Renji Hospital

\section{Zhaohui Ni}

Shanghai Jiaotong University School of Medicine Affiliated Renji Hospital

Ling Wang ( $\triangle$ Shwangling@126.com )

Shanghai Jiaotong University School of Medicine Affiliated Renji Hospital

\section{Research Article}

Keywords: ANCA-associated renal vasculitis, end-stage renal disease (ESRD), secondary infection, reduced-frequency cyclophosphamide, reduced-dose glucocorticoids.

Posted Date: October 25th, 2021

DOI: https://doi.org/10.21203/rs.3.rs-961033/v1

License: (c) (1) This work is licensed under a Creative Commons Attribution 4.0 International License. Read Full License 


\section{Abstract}

\section{Objectives}

Secondary infection caused by high-dose immunosuppressive therapy is one of the most common causes of death in patients with ANCA-associated vasculitis. This study aimed to explore whether reduced-dose immunosuppressive therapy can achieve the same efficacy while reducing secondary infection in mild to moderate ANCA-associated renal vasculitis and to determine whether early secondary infections will affect the deterioration of renal function.

\section{Methods}

The efficacy and safety of the reduced-dose regimen and standard regimen were compared using the chisquare test. The renal survival rates were estimated using the Kaplan-Meier method and compared using the log rank test. Potential variates were examined using multivariate Cox proportional hazard models to determine the risk predictors of end-stage renal disease.

\section{Results}

A total of 35 patients in the standard-dose glucocorticoid group and 23 in the reduced-dose glucocorticoid group were included. The average age of the included patients was $62.45 \pm 12.70$ years, and the baseline serum creatinine was $251.35[155.53,445] \mu \mathrm{mol} / \mathrm{L}$. Nine patients $(15.52 \%)$ developed ESRD within 24 months (7 standard vs. 2 reduced, $P=0.035$ ). Multivariate Cox regression model analysis proved that baseline serum creatinine (HR: $0.007,95 \% \mathrm{Cl}: 2.48-39.48, \mathrm{P}=0.014)$, secondary infection rate within the first 3 months (HR: 2.28, 95\% Cl: 2.14 45.27, $\mathrm{P}=0.003$ ), and persistent hematuria for more than 6 months (HR: $1.723,95 \% \mathrm{Cl}: 0.043-0.738, \mathrm{P}=0.017)$ were risk predictors of end-stage renal disease in ANCA-associated renal vasculitis.

\section{Conclusion}

The regimen of initial reduced-dose immunosuppressive therapy can significantly reduce the secondary infection rate in mild to moderate ANCA-associated renal vasculitis patients, and the efficacy is not inferior to the standard regimen. Patients who had secondary infection within the first 3 months were at higher risk of developing ESRD.

\section{Author Summary}

Conceptualization, Ziyang Li and Ling Wang; data curation, Ziyang Li; formal analysis, Ziyang Li; investigation, Ziyang Li, Jiwen Bao, Huanzhen Yao, Qin Wang, Minfang Zhang, Leyi Gu, Zhaohui Ni; original draft preparation, Ziyang Li; review and editing. Ziyang Li and Ling Wang; supervision, Ling Wang.

\section{Introduction}


Anti-neutrophil cytoplasmic antibody (ANCA)-associated vasculitis (AAV) is a group of necrotizing small vasculitis that can be divided into PR3-ANCA-positive and MPO-ANCA-positive AAV according to the serological classification ${ }^{1,2}$. Renal involvement occurs in $70 \%$ of $A A V$ patients ${ }^{3}$, especially MPO-ANCApositive $\mathrm{AAV}^{4}$, which has pauci-immune complex deposition and crescentic glomeruli as the most common renal histopathological manifestation ${ }^{5-8}$.

Although cyclophosphamide (CYC) combined with high-dose glucocorticoids has been the standard regimen in AAV as remission induction therapy for nearly five decades ${ }^{9-12}$, new immunosuppressive agents have been tried in clinical trials considering the side effects of the long-term use of cyclophosphamide and glucocorticoids ${ }^{13-15}$. For instance, rituximab is not inferior to daily oral cyclophosphamide for the induction of remission in severe ANCA-associated vasculitis and may be superior in relapsing disease, which has been proven by both RAVE and RITUXVAS trials ${ }^{3,16}$. However, due to economic reasons, rituximab is still difficult to conventionally apply in clinical practice. Additionally, there is still controversy regarding the hunting of regimens allowing for glucocorticoid reduction, which can balance efficacy and safety in patients with ANCA-associated vasculitis. Although the PEXIVAS trial was conducted to show that a rapid reduction in glucocorticoid regimen was noninferior to a standard regimen with respect to death or end-stage renal disease (ESRD) ${ }^{17,18}$, there is still no consensus on whether an initial reduced dose of glucocorticoids has similar efficacy for nonsevere diseases.

Secondary infection is the most common adverse event and the primary cause of death in immunosuppressive therapy for $A A V^{19-21}$. Currently, both elementary experiments and clinical studies have indicated that infection may trigger the formation of ANCAs and that severe cases may eventually develop into ANCA-associated vasculitis ${ }^{1,22-25}$. Therefore, the core of management of AAV is far more than achieving disease remission, and it is necessary to concentrate on reducing mortality, preventing deterioration of renal function and improving the survival quality.

This retrospective cohort study compared the efficacy and safety of reduced-frequency cyclophosphamide combined with initial reduced-dose glucocorticoids as a remission induction regimen for mild to moderate AAV patients with renal involvement. We also examined clinical variates for ESRD to determine the risk factors that can be used to recognize patients at higher risk of ESRD in the short term.

\section{Methods}

\section{Patients}

This was a single-center retrospective cohort study collecting information on 82 patients who were newly diagnosed in the Department of Nephrology, Renji Hospital, Shanghai Jiao Tong University School of Medicine from 2013 to 2020. Inclusion criteria include:1. Kidney damage caused by MPA or GPA, the conditions include at least any one of the following: $₫$ Necrotizing glomerulonephritis on biopsy; $\mathbb{R}$ Red cell casts or hematuria ( $>=30$ red cells per high power field) on urinalysis; 2 . ANCA positivity needs to be met through enzyme-linked immunosorbent assay (ELISA) positive for MPO-ANCA/PR3-ANCA or positive 
for p-ANCA/c-ANCA by indirect immunofluorescence (IIF). Exclusion criteria include: 1 . Alveolar hemorrhage or anuria囚2. Patients who had used any immunosuppressive agents for more than 2 weeks; 3. With other active autoimmune diseases at the same time, such as systemic lupus erythematosus (SLE), eosinophilic granulomatosis with polyangiitis (EGPA) or anti-glomerulus basement membrane antibody positivity; 4. Hepatitis B e antigen-positive, hepatitis $C$ antibody-positive, HIV-positive; 5 . History of malignant tumors; 6 . Pregnant or lactating women; 7. Patients who had experience with oliguria at the first visit. Finally, 58 patients meet the research conditions. (Figure 1)

\section{Treatments}

The remission-induction period was 6 months. All of the patients received intravenous pulses of cyclophosphamide (15 mg/kg), and the frequency was extended from once every 2-3 weeks according to the recommendation ${ }^{26}$ to once every 4 weeks. The maximum dose per pulse was $1 \mathrm{~g}$, adjusted for renal insufficiency. Initial prednisone or prednisolone was received at a dose of $1 \mathrm{mg}$ per kilogram per day in the standard-dose group and 0.3-0.7 mg per kilogram per day in the reduced-dose group. Patients were allowed to undergo treatment plasma exchange according to practice according to the clinician's experience. During the remission maintenance period, the frequency of cyclophosphamide switched from once every 4 weeks to once every 3 months with the same dose as before, combined with prednisone or prednisolone at a dose of 5-15 $\mathrm{mg}$ per day.

\section{Data collection}

The clinical characteristics were collected from the patients' medical records. The following characteristics were assessed at 0, 3, 6, 12, and 24 months: age, sex, presence of ANCA, anti-ANCA levels, serum creatinine(Scr) levels, estimated glomerular filtration rate (eGFR), hematuria, urinary albumin creatinine ratio(UACR),blood leukocytes, blood lymphocyte ratio, c-reaction protein(CRP),procalcitonin(PCT), Erythrocyte sedimentation rate(ESR), serum albumin, total bilirubin(TBil), glutamic-pyruvic transaminase(ALT), glutamic oxalacetic transaminase(AST), previous history(hypertension, diabetes, hyperlipemia, concomitant symptom, etc.

\section{Definitions}

Mild to moderate was defined as BVAS $₫ 18$. Renal remission was defined as stable or decreased serum creatinine, disappearance of hematuria (microscopic RBC $<10 / \mathrm{HP}$ ), and no other manifestations of AAV. Renal relapse was defined as an increase in serum creatinine (Scr) of $>30 \%$ and/or new hematuria or proteinuria. Progressive disease was defined as an elevation of serum creatinine and either the persistence of hematuria or proteinuria. ESRD was defined as the need for permanent dialysis or receipt of a renal transplant. The Birmingham Vasculitis Activity Score (BVAS) ranges from 0 to 63 , and higher scores indicate more active disease.

\section{Outcomes}


The primary end point was the occurrence of ESRD within the 24 months; the secondary end points were the rate of secondary infection and ANCA turned negative within the first 3 months and the rate of adverse events and persistent hematuria within the first 6 months.

\section{Statistical analysis}

SPSS 22.0 statistical software was used for statistical analysis. If measurement data conformed to a normal distribution, they were expressed as the mean \pm standard deviation $(x \pm s)$, and the two-sample $t$ test was used for comparison between the two groups. If not, they were expressed as the median (interquartile range) $[\mathrm{M}(\mathrm{P} 25, \mathrm{P} 75)]$, and the Mann-Whitney $\mathrm{U}$ test was used for comparison between the two groups. Categorical data were expressed as a number (proportion), and the comparison between two groups of unordered classification data used the chi-square test. The renal survival rate was estimated using the Kaplan-Meier method and compared using the log rank test. Factors with a P value $<0.05$ in Kaplan-Meier analysis were included in multivariate Cox proportional hazard models to identify the predictors of ESRD. Receiver operating characteristic (ROC) curve analysis was used to determine the predictive performance. The area under the curve (AUC) was calculated as the diagnostic measure of the test. A P-value $<0.05$ was considered to denote statistically significant differences.

\section{Results}

\section{Patient characteristics}

A total of 82 patients with ANCA-associated renal vasculitis diagnosed in the Department of Nephrology, Renji Hospital, Shanghai Jiaotong University School of Medicine from January 2013 to September 2020 were included. Twenty-four people who did not meet the inclusion criteria were excluded, and 58 people were involved in this study. Among them, 35 were in the standard-dose glucocorticoid group, and 23 were in the reduced-dose glucocorticoid group. The ratio of males to females among 58 people was 16/42, the average age was $62.45 \pm 12.70$ years, the ratio of PR3-ANCA to MPO-ANCA was 5/53, the baseline Scr was $251.35[155.53,445] \mu \mathrm{mo} / \mathrm{L}$, and 42 patients underwent renal biopsy. The median follow-up time was 17 $[7.5,31.25]$ months, and the cumulative cyclophosphamide dose was $1.2[0.6,1.6] \mathrm{g}$ at 3 months and was $2.4[1.6,3.7] \mathrm{g}$ at 6 months in all included patients regardless of the dose of glucocorticoids.

\section{Primary outcomes}

A total of 9 patients developed ESRD within 24 months, 7 patients in the standarddose glucocorticoid group and 2 patients in the reduced-dose glucocorticoid group $(P=0.25)$ (figure 2). Of these nine patients, all were positive for MPO-ANCA, with a baseline Scr of $460.22 \pm 141.20 \mu \mathrm{mol} / \mathrm{L}$, baseline UACR 796[523.2,1753.1] mg/g, BVSA score 12[12,17], 2/9 cases complicated with underlying pulmonary diseases (one had pulmonary tuberculosis history, another had chronic obstructive pulmonary disease); 6/9 patients developed pulmonary infections within 3 months, and 4/9 patients had persistent hematuria for more than 6 months. The proportion of patients who developed ESRD in the standard- 
dose glucocorticoid group was significantly higher than that in the reduced-dose glucocorticoid group, but there was no significant difference due to the limited sample size. (Table 1)

\section{Secondary end points}

Ten patients (17.24\%) developed infection within the first 3 months, out of 10 patients, 1 patient developed upper respiratory tract infection, 1 patient developed urinary tract infection, 1 patient developed cryptococcal infection, and the remaining 7 patients developed pulmonary bacterial infections. There were $9 / 10$ patients $(25.71 \%)$ in the standard-dose group, of whom $1 / 9$ developed cryptococcal infection, and 1/10 developed cryptococcal infection (4.35\%) in the reduced-dose group $(P=0.035)$. The secondary infection rate in the reduced-dose group was significantly lower than that of the standard-dose group. Twenty-eight/58 patients (48.28\%) with ANCAs became negative within the first 3 months, $18 / 35$ patients $(51.43 \%)$ in the standard-dose group, and $10 / 23$ patients $(43.48 \%)$ in the reduced-dose group $(P=0.553)$. There was no significant difference between the groups in the rate of ANCA conversion. (Table 1)

A total of 19 adverse events occurred in 17 patients within the first six months: 13 patients had pulmonary infection ( 2 of 13 patients had cryptococcal infection, and the others had bacterial infections; 1 of 13 patients simultaneously had elevated transaminase, and 1 of 13 patients simultaneously had central serous chorioretinopathy), 2 patients had herpes, 1 patient had urinary tract infection, and 1 patient had upper respiratory tract infection. Fortyfive/58 patients $(77.57 \%)$ had disappeared hematuria within 6 months, $26 / 35$ patients $(74.29 \%)$ in the standard-dose group, and 19/23 patients (82.61\%) in the reduced-dose group $(P=0.46)$. (Table 1)

\section{Risk predictors of ESRD}

Age, baseline Scr, infection, Scr drop rate and ANCA became negative within the first 3 months, persistent hematuria for more than 6 months, baseline UACR, baseline albumin, baseline lymphocyte ratio, hypertension, diabetes, and chronic pulmonary disease were included in the Kaplan-Meier analysis (Figure 3-7), and the renal survival rate was compared by the log rank test (Table 2). Among them, the baseline Scr, infection and Scr drop rate within the first 3 months, persistent hematuria for more than 6 months, and baseline UACR were significantly correlated with endpoint events with $P$ values less than 0.05 and were included in the multivariate Cox proportional hazard model. The results showed that baseline Scr[HR 1.008, 95\% CI[1.001, 1.014], $\mathrm{P}=0.014]$, infection within first 3 months[HR 9.835, $95 \% \mathrm{Cl}[2.137,45.270], \mathrm{P}=0.003]$, and persistent hematuria for more than 6 months[HR 5.603,95\% $\mathrm{Cl}[1.355$, 23.181], $P=0.017$ ] were risk predictors of ESRD, there is no significant relationship between baseline UACR, baseline lymphocyte ratio, combined hypertension, diabetes, chronic pulmonary disease and ESRD (Table 3). ROC curves were designed to prove the value of different factors in predicting the primary outcome. The AUCs for baseline Scr, infection within the first 3 months, and persistent hematuria were $0.82(\mathrm{P}=0.002,95 \% \mathrm{Cl} 0.71-0.93), 0.79(\mathrm{P}=0.00695 \% \mathrm{Cl} 0.60-0.98)$ and $0.565(\mathrm{P}=0.541,95 \% \mathrm{Cl} 0.35-0.78)$. (Figure 8). 


\section{Discussion}

The AAV patients in our study were those with BVAS scores less than 18, all of whom had no alveolar hemorrhage or anuria. Since most of the current studies focused on severe cases of AAV, the optimal treatment of mild to moderate patients is worthy of further discussion.

Multiple studies demonstrated that the most common cause of death in patients with ANCA-associated vasculitis was secondary infection caused by high-dose immunosuppressive therapy, very few due to active vasculitis. The RITUXVAS trial indicated that most deaths occur in the early stages of treatment (within 3 months), $50 \%$ of which resulted from secondary infection ${ }^{3}$. A clinical study from China also found that secondary infection was the leading cause of death through a one-year follow-up of AAV patients ${ }^{19-21}$. The hazard factors associated with severe infection in AAV patients include the use of chronic immunosuppression, long-term placement of central intravenous catheters, uraemia/dialysis dependency, and gamma globulin depletion caused by plasma exchange ${ }^{27}$; in particular, the use of highdose glucocorticoids and cyclophosphamide was an independent risk factor for infection in AAV patients ${ }^{26,28,29}$. Consequently, based on the presupposition of achieving remission, a reduced regimen of immunosuppressive therapy can be considered an option to lower the infection rate, especially in patients with mild to moderate ANCA-associated renal vasculitis. The frequency of intravenous pulses of cyclophosphamide and the initial dose of prednisone or prednisolone were both reduced in our study, considering some factors as follows: most patients with AAV have no or mild extrarenal manifestations, some concomitant infection at the first visit and cyclophosphamide itself has certain nephrotoxicity.

The secondary infection of AAV patients is not only the main cause of death during the course of the disease but also may be an important factor in aggravating the condition. A large number of elementary experimental and clinical studies have explored the causal relationship between infection and vasculitis. Elementary experimental studies have summarized some proposed mechanisms of ANCA formation during the process of infection as follows: initiation of autoimmune response by microbial peptides that are complementary to auto antigens; epigenetic silencing and antigen complementarity leading to upregulation of autoantigen genes; molecular mimicry between bacterial and self-antigens; formation of neutrophil extracellular traps that stimulate immune processes including production of ANCA; and interaction of bacterial components with Toll-like receptors, which leads to formation of mediators affecting the immune responses to infections and can trigger ANCA production ${ }^{22-25}$. Clinical studies have shown that chronic nasal Staphylococcus aureus carriage in both GPA and MPA patients has a higher risk of relapse, especially GPA patients, who have an 8-fold increase in the risk of relapse, and there is also evidence suggesting that antibacterial treatment may reduce the risk of relapse in patients with sinusitis ${ }^{30-35}$; simultaneously, persistent infection of Chlamydia pneumoniae has been proven to contribute to the pathogenesis of MPO-ANCA-associated renal vasculitis in a process that elicits a hypersensitivity reaction of the host and ultimately results in strongly enhanced focal inflammatory reaction and necrotizing vasculitis ${ }^{36-38}$. In our study, the data showed that the infection rate within the first 3 months after treatment was significantly different between the two groups. Furthermore, the Cox 
proportional hazard models demonstrated that AAV patients who had infection within the first 3 months had a lower renal survival rate, and the results of the area under the curve (AUC) analysis demonstrated the predictive value of this variable.

In addition to infection, the multivariate Cox regression model showed that patients with higher baseline creatinine and persistent hematuria for more than 6 months were also at higher risk of developing ESRD in the early stages of the disease. In AAV patients, microscopic hematuria is a marker of renal damage and disease activity, but the significance is still controversial. Although previous studies have indicated that persistent hematuria can be used as an important predictor of future renal relapse in AAV patients after induction therapy ${ }^{39,40}$, other studies showed that persistent hematuria only indicates renal damage degree and cannot predict the progression of the disease ${ }^{41}$. A retrospective study demonstrated that no disappearance of hematuria for more than 90 days following standard treatment after diagnosis of ANCA-associated renal vasculitis was not related to a lower eGFR at 1 year of follow-up ${ }^{42}$. However, in our study, with a median follow-up time of 17 months, AAV patients with persistent hematuria for more than 6 months were at higher risk of developing ESRD. A small series clinical trial has given us some mentality. This trial involved nine AAV patients in clinical remission with either persistent or new hematuria, and given them repeat kidney biopsies, the results found various histopathologic types, including other types of active glomerulus nephritis ${ }^{43}$, which indicates that patients with persistent hematuria may have new renal pathological manifestations on the basis of the original disease, which would affect the prognosis. In summary, we believe that in the process of immunosuppressive treatment, persistent hematuria may represent the degree of renal damage and may also have a certain predictive value for the prognosis of the disease. For patients with persistent hematuria, clinicians should promptly pay attention to and adjust the treatment strategy to prolong the occurrence of ESRD.

However, our study is a retrospective study with a limited sample size, and its results need to be further confirmed in a larger prospective cohort study. The patients included in this study were mainly MPA patients; therefore, the results may not be applicable to GPA and EGPA patients. The median follow-up time in this study was 17 months, which could be longer in subsequent studies.

In conclusion, our study proved that a decrease in the secondary infection rate would lower mortality in the early stage and may prevent the progression or relapse of disease, thereby delaying the deterioration of kidney function. Hence, the regimen of reduced-frequency cyclophosphamide combined with initial reduced-dose glucocorticoids may be more appropriate than the standard-dose regimen in mild to moderate AAV and can be used as an option. In addition, we propose that patients with higher baseline serum creatinine, secondary infection within the first 3 months, and persistent hematuria for more than 6 months of treatment were at higher risk of developing ESRD.

\section{Declarations}

\section{Ethics approval and consent to participate}


The study have been performed in accordance with the Declaration of Helsinki and was approved by the Ethics Committee of Renji Hospital, and due to the retrospective nature of the study, the need for patients' informed consent was waived.

\section{Consent for publication}

Not applicable.

\section{Availability of data and materials}

\section{All data generated or analysed during this study are included in this published article.}

\section{Competing interests}

The authors report no conflicts of interest. The authors alone are responsible for the content and writing of the article.

\section{Funding}

None.

\section{Authors' contributions}

Conceptualization, Ziyang Li and Ling Wang; data curation, Ziyang Li; Formal analysis, Ziyang Li; investigation, Ziyang Li, Jiwen Bao, Huanzhen Yao, Minfang Zhang, Leyi Gu, Zhaohui Ni; original draft preparation, Ziyang Li; review and editing. Ziyang Li and Ling Wang; supervision, Ling Wang.

\section{Acknowledgements}

Not applicable.

\section{References}

1. Li ZY, Ma TT, Chen M, Zhao MH. The Prevalence and Management of Anti-Neutrophil Cytoplasmic Antibody-Associated Vasculitis in China. Kidney diseases (Basel, Switzerland) 2016;1:216-23.

2. Jennette JC, Falk RJ, Bacon PA, et al. 2012 revised International Chapel Hill Consensus Conference Nomenclature of Vasculitides. Arthritis and rheumatism 2013;65:1-11.

3. Jones RB, Tervaert JW, Hauser T, et al. Rituximab versus cyclophosphamide in ANCA-associated renal vasculitis. The New England journal of medicine 2010;363:211-20.

4. Millet A, Pederzoli-Ribeil M, Guillevin L, Witko-Sarsat V, Mouthon L. Antineutrophil cytoplasmic antibody-associated vasculitides: is it time to split up the group? Annals of the rheumatic diseases 2013;72:1273-9. 
5. Kallenberg CG. Key advances in the clinical approach to ANCA-associated vasculitis. Nature reviews Rheumatology 2014;10:484-93.

6. Kallenberg CG, Stegeman CA, Abdulahad WH, Heeringa P. Pathogenesis of ANCA-associated vasculitis: new possibilities for intervention. American journal of kidney diseases : the official journal of the National Kidney Foundation 2013;62:1176-87.

7. Sinico RA, Di Toma L, Radice A. Renal involvement in anti-neutrophil cytoplasmic autoantibody associated vasculitis. Autoimmunity reviews 2013;12:477-82.

8. Berden AE, Ferrario F, Hagen EC, et al. Histopathologic classification of ANCA-associated glomerulonephritis. Journal of the American Society of Nephrology : JASN 2010;21:1628-36.

9. de Groot K, Harper L, Jayne DR, et al. Pulse versus daily oral cyclophosphamide for induction of remission in antineutrophil cytoplasmic antibody-associated vasculitis: a randomized trial. Annals of internal medicine 2009;150:670-80.

10. Jayne D, Rasmussen N, Andrassy K, et al. A randomized trial of maintenance therapy for vasculitis associated with antineutrophil cytoplasmic autoantibodies. The New England journal of medicine 2003;349:36-44.

11. Jayne DR, Gaskin G, Rasmussen N, et al. Randomized trial of plasma exchange or high-dosage methylprednisolone as adjunctive therapy for severe renal vasculitis. Journal of the American Society of Nephrology : JASN 2007;18:2180-8.

12. Mukhtyar $C$, Guillevin L, Cid MC, et al. EULAR recommendations for the management of primary small and medium vessel vasculitis. Annals of the rheumatic diseases 2009;68:310-7.

13. Jayne D. Challenges in the management of microscopic polyangiitis: past, present and future. Current opinion in rheumatology 2008;20:3-9.

14. Koukoulaki $\mathrm{M}$, latrou $\mathrm{C}$. The role of mycophenolate in the treatment of antineutrophil cytoplasmic antibody-associated vasculitis. World journal of nephrology 2019;8:75-82.

15. Faurschou M, Westman K, Rasmussen N, et al. Brief Report: long-term outcome of a randomized clinical trial comparing methotrexate to cyclophosphamide for remission induction in early systemic antineutrophil cytoplasmic antibody-associated vasculitis. Arthritis and rheumatism 2012;64:3472-7.

16. Stone JH, Merkel PA, Spiera R, et al. Rituximab versus cyclophosphamide for ANCA-associated vasculitis. The New England journal of medicine 2010;363:221-32.

17. Pepper RJ, McAdoo SP, Moran SM, et al. A novel glucocorticoid-free maintenance regimen for antineutrophil cytoplasm antibody-associated vasculitis. Rheumatology (Oxford, England) 2019;58:2608.

18. Walsh M, Merkel PA, Peh CA, et al. Plasma Exchange and Glucocorticoids in Severe ANCA-Associated Vasculitis. The New England journal of medicine 2020;382:622-31.

19. Little MA, Nightingale P, Verburgh CA, et al. Early mortality in systemic vasculitis: relative contribution of adverse events and active vasculitis. Annals of the rheumatic diseases 2010;69:1036-43. 
20. Lai QY, Ma TT, Li ZY, Chang DY, Zhao MH, Chen M. Predictors for mortality in patients with antineutrophil cytoplasmic autoantibody-associated vasculitis: a study of 398 Chinese patients. The Journal of rheumatology 2014;41:1849-55.

21. Li ZY, Gou SJ, Chen M, Zhao MH. Predictors for outcomes in patients with severe ANCA-associated glomerulonephritis who were dialysis-dependent at presentation: a study of 89 cases in a single Chinese center. Seminars in arthritis and rheumatism 2013;42:515-21.

22. Haris Á, Polner K, Arányi J, et al. Simple, readily available clinical indices predict early and late mortality among patients with ANCA-associated vasculitis. BMC nephrology 2017;18:76.

23. Hutton HL, Holdsworth SR, Kitching AR. ANCA-Associated Vasculitis: Pathogenesis, Models, and Preclinical Testing. Seminars in nephrology 2017;37:418-35.

24. Konstantinov KN, Ulff-Møller CJ, Tzamaloukas AH. Infections and antineutrophil cytoplasmic antibodies: triggering mechanisms. Autoimmunity reviews 2015;14:201-3.

25. Nakazawa D, Shida H, Tomaru U, et al. Enhanced formation and disordered regulation of NETs in myeloperoxidase-ANCA-associated microscopic polyangiitis. Journal of the American Society of Nephrology : JASN 2014;25:990-7.

26. Yates $M$, Watts RA, Bajema IM, et al. EULAR/ERA-EDTA recommendations for the management of ANCA-associated vasculitis. Annals of the rheumatic diseases 2016;75:1583-94.

27. Charlier C, Henegar C, Launay 0 , et al. Risk factors for major infections in Wegener granulomatosis: analysis of 113 patients. Annals of the rheumatic diseases 2009;68:658-63.

28. Schönermarck U, Gross WL, de Groot K. Treatment of ANCA-associated vasculitis. Nature reviews Nephrology 2014;10:25-36.

29. Goupil R, Brachemi S, Nadeau-Fredette AC, et al. Lymphopenia and treatment-related infectious complications in ANCA-associated vasculitis. Clinical journal of the American Society of Nephrology : CJASN 2013;8:416-23.

30. Popa ER, Stegeman CA, Abdulahad WH, et al. Staphylococcal toxic-shock-syndrome-toxin-1 as a risk factor for disease relapse in Wegener's granulomatosis. Rheumatology (Oxford, England) 2007;46:1029-33.

31. Popa ER, Stegeman CA, Bos NA, Kallenberg CG, Tervaert JW. Staphylococcal superantigens and T cell expansions in Wegener's granulomatosis. Clinical and experimental immunology 2003;132:496504.

32. Stegeman CA, Tervaert JW, de Jong PE, Kallenberg CG. Trimethoprim-sulfamethoxazole (cotrimoxazole) for the prevention of relapses of Wegener's granulomatosis. Dutch Co-Trimoxazole Wegener Study Group. The New England journal of medicine 1996;335:16-20.

33. Stegeman CA, Tervaert JW, Sluiter WJ, Manson WL, de Jong PE, Kallenberg CG. Association of chronic nasal carriage of Staphylococcus aureus and higher relapse rates in Wegener granulomatosis. Annals of internal medicine 1994;120:12-7.

34. Tanaka H, Yamaguchi M, Katsuno T, et al. Association between sinusitis and relapse and changes in the myeloperoxidase-antineutrophil cytoplasmic antibody in microscopic polyangiitis. PloS one 
2020;15:e0243572.

35. Zycinska K, Wardyn KA, Zielonka TM, Demkow U, Traburzynski MS. Chronic crusting, nasal carriage of Staphylococcus aureus and relapse rate in pulmonary Wegener's granulomatosis. Journal of physiology and pharmacology : an official journal of the Polish Physiological Society 2008;59 Suppl 6:825-31.

36. Campbell LA, Kuo CC. Chlamydia pneumoniae and atherosclerosis. Seminars in respiratory infections 2003;18:48-54.

37. Kallenberg CG. Autoantibodies to myeloperoxidase: clinical and pathophysiological significance. Journal of molecular medicine (Berlin, Germany) 1998;76:682-7.

38. van Zandbergen G, Gieffers J, Kothe $\mathrm{H}$, et al. Chlamydia pneumoniae multiply in neutrophil granulocytes and delay their spontaneous apoptosis. Journal of immunology (Baltimore, Md : 1950) 2004;172:1768-76.

39. Kemna MJ, Damoiseaux J, Austen J, et al. ANCA as a predictor of relapse: useful in patients with renal involvement but not in patients with nonrenal disease. Journal of the American Society of Nephrology : JASN 2015;26:537-42.

40. Rhee RL, Davis JC, Ding L, et al. The Utility of Urinalysis in Determining the Risk of Renal Relapse in ANCA-Associated Vasculitis. Clinical journal of the American Society of Nephrology : CJASN 2018;13:251-7.

41. Magrey MN, Villa-Forte A, Koening CL, Myles JL, Hoffman GS. Persistent hematuria after induction of remission in Wegener granulomatosis: a therapeutic dilemma. Medicine 2009;88:315-21.

42. Chen TK, Murakami C, Manno RL, Geetha D. Hematuria duration does not predict kidney function at 1 year in ANCA-associated glomerulonephritis. Seminars in arthritis and rheumatism 2014;44:198-201.

43. Geetha D, Seo P, Ellis C, Kuperman M, Levine SM. Persistent or new onset microscopic hematuria in patients with small vessel vasculitis in remission: findings on renal biopsy. The Journal of rheumatology 2012;39:1413-7.

\section{Tables}

Table 1. Clinical characteristics of $58 \mathrm{AAV}$ patients with renal involvement. 


\section{All patients \\ standard-dose \\ reduced-dose \\ P \\ glucocorticoids group glucocorticoids group value}

\section{Baseline characteristics}

\begin{tabular}{|c|c|c|c|c|}
\hline Number of patients & 58 & 35 & 23 & \\
\hline Age & $62.45 \pm 12.70$ & $61.14 \pm 12.19$ & $64.44 \pm 13.47$ & 0.92 \\
\hline Sex (female/male) & $42 / 16$ & $25 / 10$ & $17 / 6$ & 0.84 \\
\hline MPA/GPA & $53 / 5$ & $34 / 1$ & $19 / 4$ & 0.052 \\
\hline BVAS & $13.24 \pm 2.15$ & $13.00 \pm 2.02$ & $13.61 \pm 2.31$ & 0.20 \\
\hline $\operatorname{Scr}(\mu \mathrm{mol} / \mathrm{L})$ & $251[156,445]$ & $285[164,463]$ & $210[152,346]$ & 0.29 \\
\hline UACR (mg/g) & $875[514,1404]$ & $868[586,1338]$ & $878[376,1723]$ & 0.79 \\
\hline Albumin $<=25 \mathrm{~g} / \mathrm{L}$ & $2 / 58$ & $0 / 35$ & $2 / 23$ & 0.08 \\
\hline $\begin{array}{l}\text { Lymphocyte } \\
\text { ratio<=10\% }\end{array}$ & $10 / 58$ & $6 / 35$ & $4 / 23$ & 0.98 \\
\hline CRP (mg/L) & $8.1[2.4,35.6]$ & $5.4[2.4,27.1]$ & $13[0.7,46.0]$ & 0.29 \\
\hline $\operatorname{ESR}(\mathrm{mm} / \mathrm{h})$ & $78 \pm 34$ & $84 \pm 33$ & $68 \pm 34$ & 0.74 \\
\hline \multicolumn{5}{|l|}{ Organ involvement } \\
\hline Fever & $7 / 58$ & $4 / 35$ & $3 / 23$ & 0.85 \\
\hline Arthralgia & $6 / 58$ & $4 / 35$ & $2 / 23$ & 0.74 \\
\hline $\begin{array}{l}\text { Loss of weight at } \\
\text { least } 2 \mathrm{~kg}\end{array}$ & $4 / 58$ & $1 / 35$ & $3 / 23$ & 0.13 \\
\hline $\begin{array}{l}\text { Mucous membranes } \\
\text { and eyes }\end{array}$ & $1 / 58$ & $1 / 35$ & $0 / 23$ & 0,41 \\
\hline Lung infiltrate & $9 / 58$ & $4 / 35$ & $5 / 23$ & 0.28 \\
\hline Pleural effusion & $4 / 58$ & $2 / 35$ & $2 / 23$ & 0.66 \\
\hline \multicolumn{5}{|l|}{ Medical history } \\
\hline Hypertension & $39 / 58$ & $24 / 35$ & $15 / 23$ & 0.79 \\
\hline Diabetes & $12 / 58$ & $9 / 35$ & $3 / 23$ & 0.24 \\
\hline $\begin{array}{l}\text { Chronic pulmonary } \\
\text { disease }\end{array}$ & $10 / 58$ & $4 / 35$ & $6 / 23$ & 0.06 \\
\hline $\begin{array}{l}\text { Follow-up time } \\
\text { (month) }\end{array}$ & $17[7.50,31.25]$ & $25[9,39]$ & $12[6,24]$ & 0.10 \\
\hline
\end{tabular}

\section{Cumulative amount of $\mathrm{CYC}(\mathrm{g})$}


At the 3th month

At the 6th month

Outcome at 24 month

ESRD

$9 / 58(15.52 \%)$

$7 / 35(20 \%)$

$2 / 23(8.7 \%)$

0.25

\section{Outcome at 3 month}

\begin{tabular}{llllc}
\hline Remission rate & $\begin{array}{l}49 / 58 \\
(84.48 \%)\end{array}$ & $30 / 35(85.71 \%)$ & $19 / 23(82.61 \%)$ & 0.75 \\
\hline Infection rate & $10 / 58(17.24 \%)$ & $9 / 35(25.72 \%)$ & $1 / 23(4.35 \%)$ & 0.035 \\
\hline Scr drop rate & $28 \%[9 \%, 43 \%]$ & $27 \%[15 \%, 42 \%]$ & $28 \%[2 \%, 51 \%]$ & 0.70 \\
\hline Negative ANCA rate & $28 / 58(48.28 \%)$ & $18 / 35(51.43 \%)$ & $10 / 23(43.48 \%)$ & 0.55 \\
\hline Outcome at 6 month & & & & \\
\hline Persistent hematuria & $13 / 58(22.31 \%)$ & $9 / 35(25.71 \%)$ & $4 / 23(17.39 \%)$ & 0.46 \\
\hline Remission rate & $50 / 58(86.21 \%)$ & $29 / 35(82.86 \%)$ & $21 / 23(91.30 \%)$ & 0.36 \\
\hline Table 1. (continued) & & & & \\
\hline Infection rate & $15 / 58(25.86 \%)$ & $10 / 35(28.57 \%)$ & $5 / 23(21.74 \%)$ & 0.56 \\
\hline Adverse events & $17 / 58(29.31 \%)$ & $10 / 35(28.57 \%)$ & $7 / 23(30.43 \%)$ & 0.88
\end{tabular}

Data conforming to a normal distribution are expressed as the mean \pm standard deviation $(x \pm s)$, data that did not conform to a normal distribution are expressed as the median (interquartile range) [M(P25, P75)], and categorical data are expressed as a number proportion. Abbreviations: MPA, microscopic polyangiitis; GPA, granulomatosis with polyangiitis; BVAS, Birmingham vasculitis activity score; Scr, serum creatinine; UACR, urinary albumin creatinine ratio; ESRD, end-stage renal disease; CRP, Creactive protein; ESR, erythrocyte sedimentation rate.

\section{Table 2. Kaplan-Meier analysis}




\begin{tabular}{ll} 
Factors & Log Rank test P value \\
\hline Baseline Scr & 0.013 \\
\hline Infection within 3 months & 0.00004 \\
\hline Persistent hematuria within 6 months & 0.003 \\
\hline BVAS>=15 & 0.016 \\
\hline UACR $>3500 \mathrm{mg} / \mathrm{g}$ & 0.015 \\
\hline ANCA-negative within 3 months & 0.692 \\
\hline Age>=65 years & 0.891 \\
\hline Albumin<=25g/L & 0.606 \\
\hline Lymphocyte ratio<=10\% & 0.799 \\
\hline Hypertension & 0.459 \\
\hline Diabetes & 0.383 \\
\hline Chronic pulmonary disease & 0.536
\end{tabular}

The Kaplan-Meier method was used to estimate the cumulative probability of renal survival rate, and the log rank test was used for comparison. A P-value $<0.05$ was considered to denote statistically significant differences.

Table 3. Multivariate COX regression analysis

\begin{tabular}{|llll|}
\hline Factors & HR & $95 \% \mathrm{Cl}$ & $\mathrm{PI}$ \\
\hline Baseline Scr & 1.008 & {$[1.001,1.014]$} & 0.014 \\
\hline Infection within 3 months & 9.835 & {$[2.137,45.270]$} & 0.003 \\
\hline Persistent hematuria within 6 months & 5.603 & {$[1.355,23.181]$} & 0.017 \\
\hline BVAS $>=\mathbf{1 5}$ & $/$ & $/$ & 0.759 \\
\hline UACR $>\mathbf{3 5 0 0} \mathbf{m g} / \mathbf{g}$ & $/$ & $/$ & 0.178 \\
\hline
\end{tabular}

Factors with a $\mathrm{P}$ value $<0.05$ in Kaplan-Meier analysis were included in multivariate Cox proportional hazard models. A P-value $<0.05$ was considered to denote statistically significant differences.

\section{Figures}




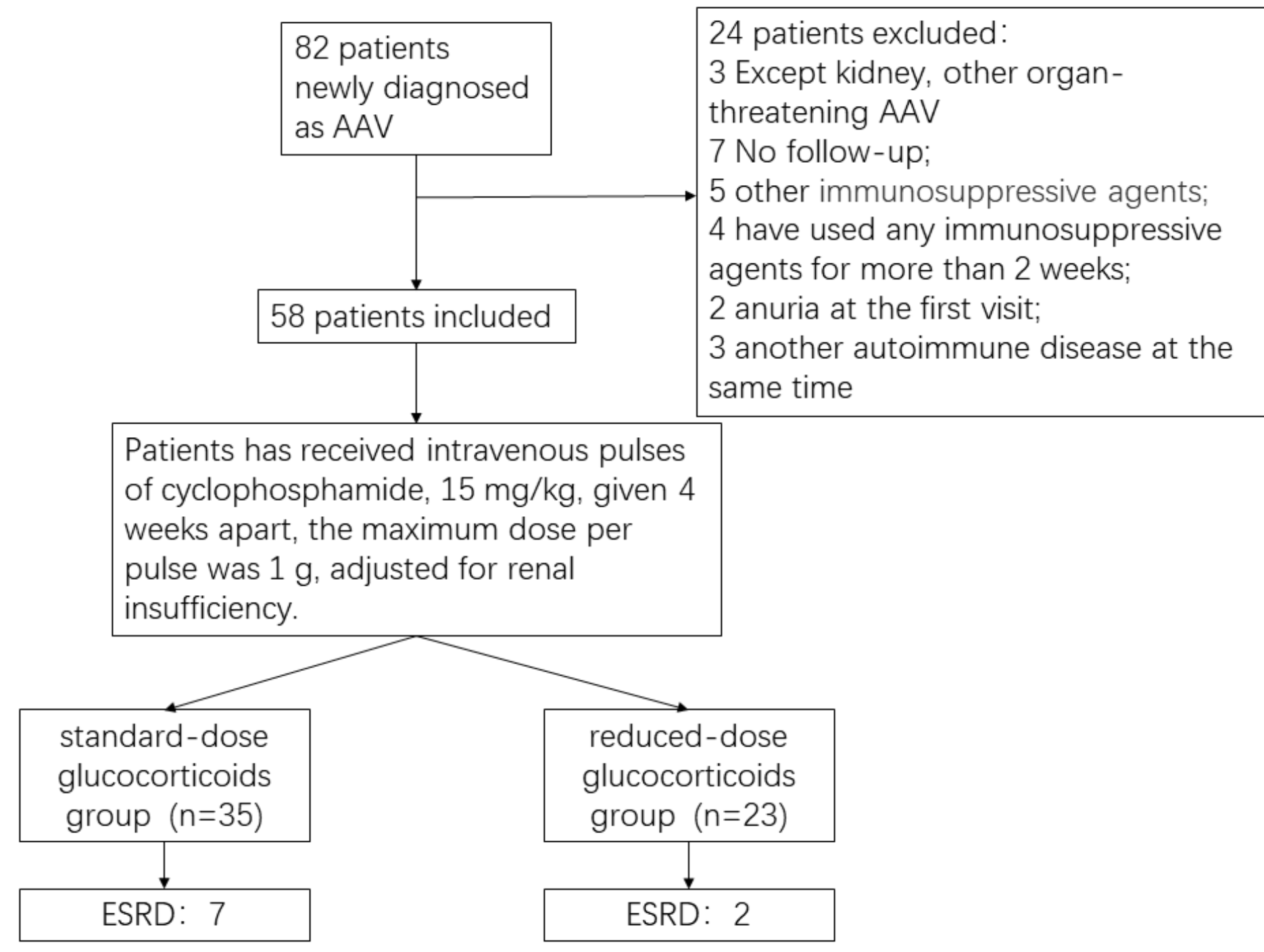

\section{Figure 1}

A total of 82 patients with ANCA-related vasculitis were included. Twenty-four patients who did not meet the inclusion criteria were excluded, and a total of 58 patients were involved in this study. All patients received intravenous pulses of cyclophosphamide, $15 \mathrm{mg} / \mathrm{kg}$, given 4 weeks apart, and the maximum dose per pulse was $1 \mathrm{~g}$, adjusted for renal insufficiency. Thirty-five patients received prednisone or prednisolone at a dose of $1 \mathrm{mg}$ per kilogram per day in the standard-dose group, and 23 patients received prednisone or prednisolone at a dose of 0.3-0.7 mg per kilogram per day in the reduced-dose group. A total of 9 patients developed ESRD, 7 patients in the standard-dose glucocorticoid group and 2 patients in the reduced-dose glucocorticoid group. 


\section{standard-dose glucocorticoids group reduced-dose glucocorticoids group}

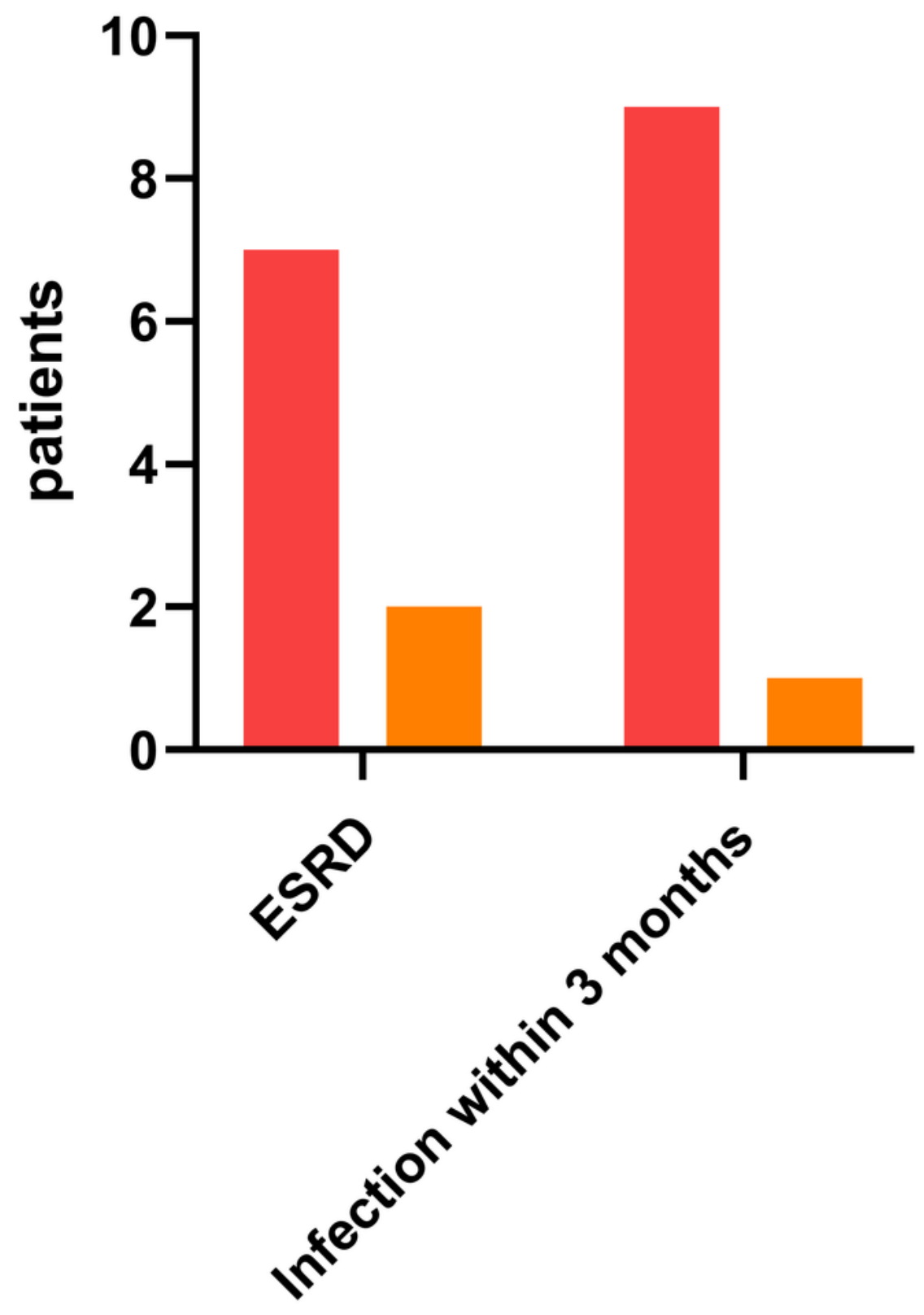

Figure 2

A total of 9 patients developed ESRD, 7 patients in the standard-dose glucocorticoid group and 2 patients in the reduced-dose glucocorticoid group $(P=0.25)$. Ten patients $(17.24 \%)$ developed infection within the first 3 months: 9 patients $(25.71 \%)$ in the standard-dose group and 1 patient $(4.35 \%)$ in the reduced-dose group $(P=0.035)$. 


\section{Baseline Scr}

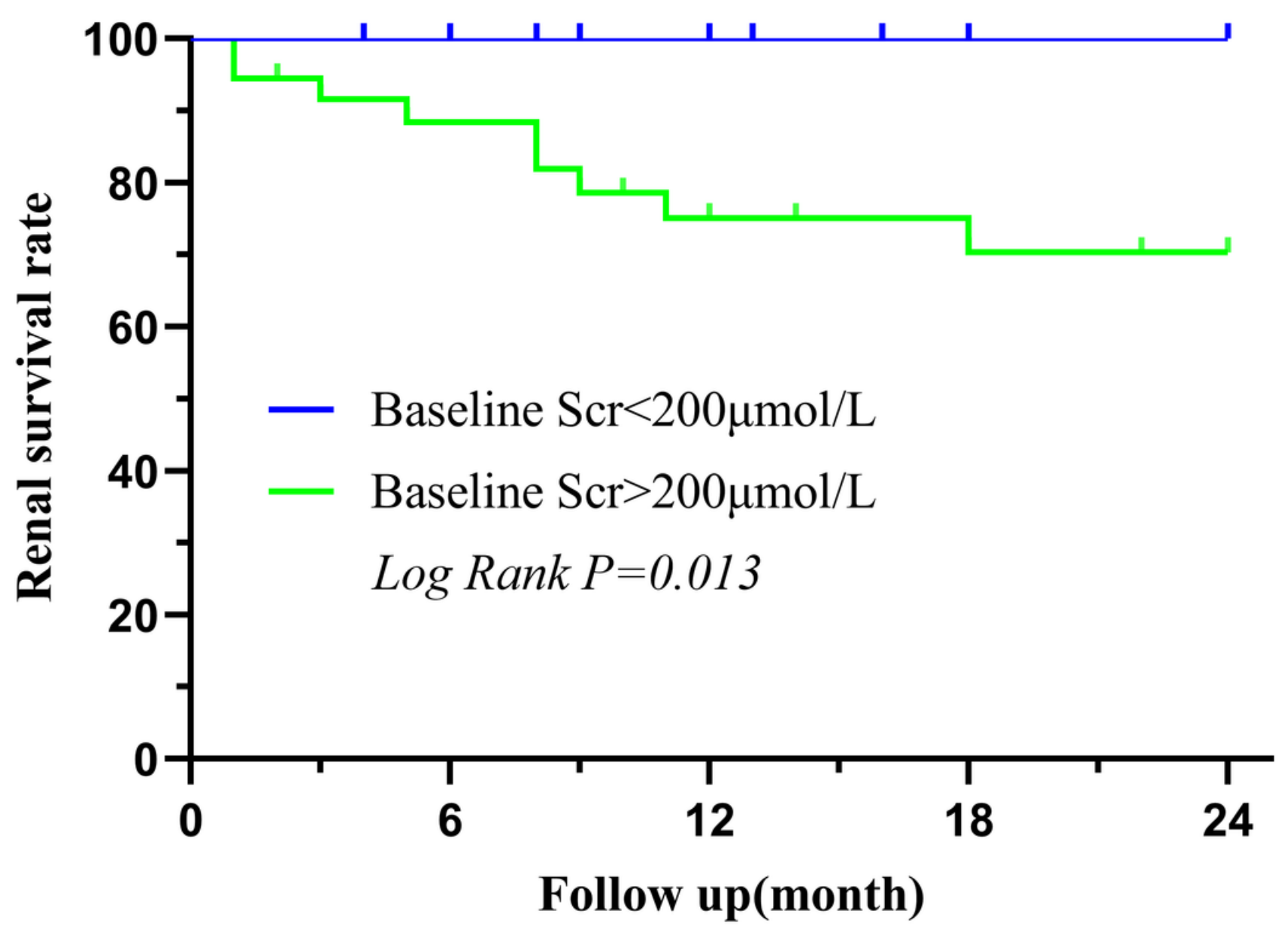

Figure 3

Comparison of the renal survival rate of patients with baseline $S c r \geq 200 \mu \mathrm{mol} / \mathrm{L}$ and patients with baseline Scr<200 $\mu \mathrm{mol} / \mathrm{L}$. 


\section{Peresistent hematuria}

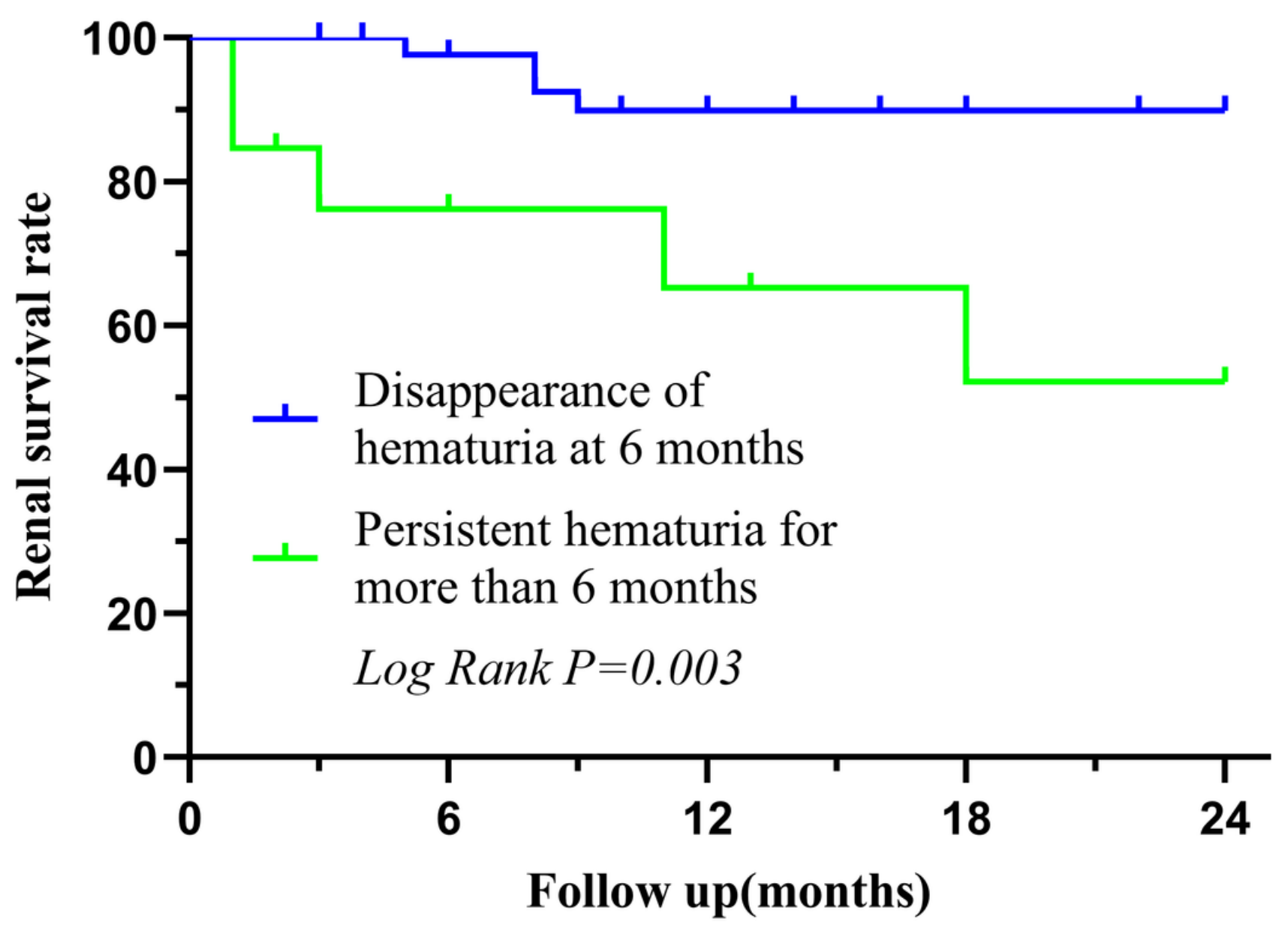

Figure 4

Comparison of the renal survival rate of patients who developed infection within 3 months and those who did not develop infection within 3 months. 


\section{Infection within 3 months}

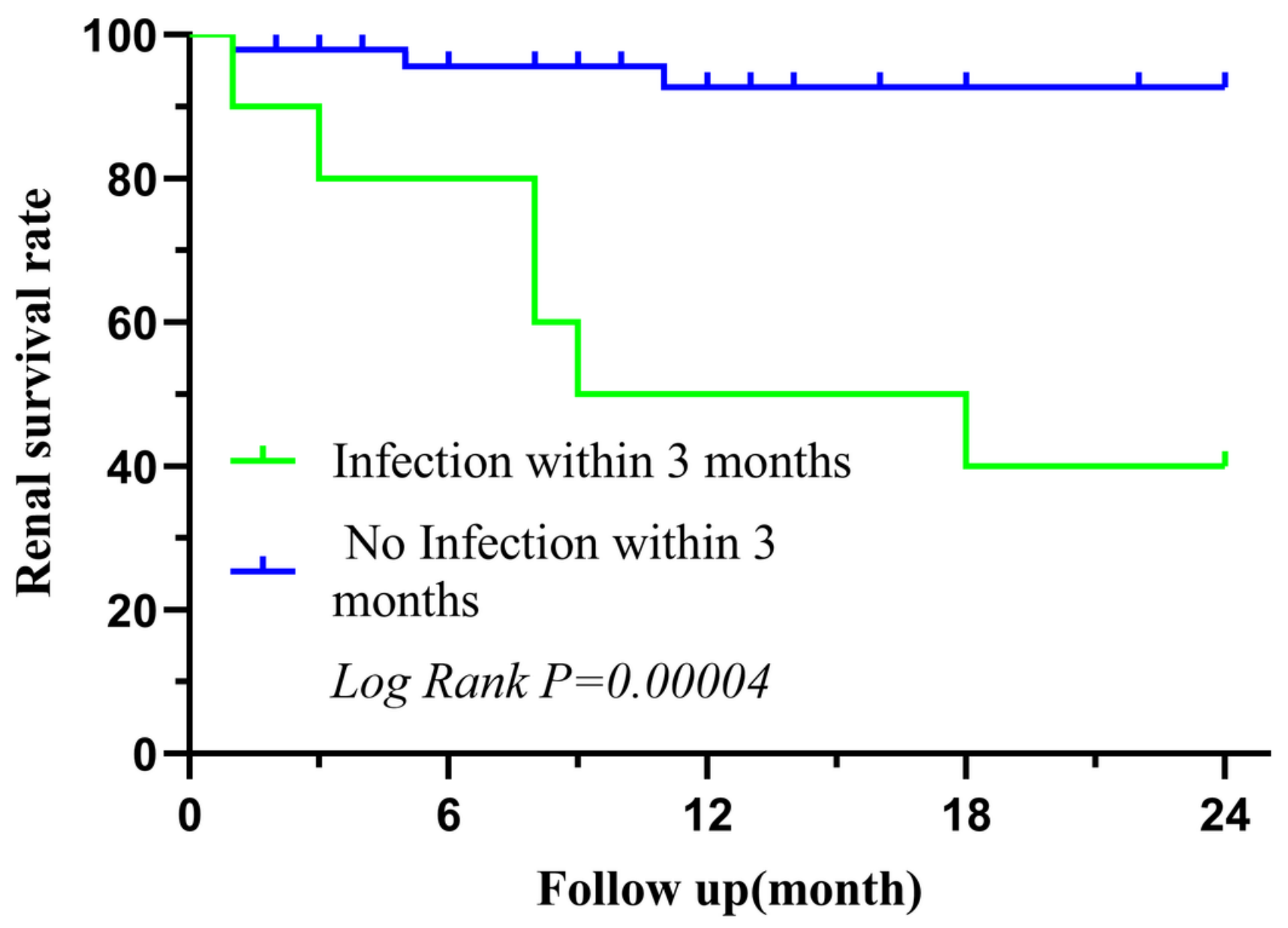

Figure 5

Comparison of the renal survival rate of patients with persistent hematuria at 6 months and those with disappearance of hematuria at 6 months. 
BVAS

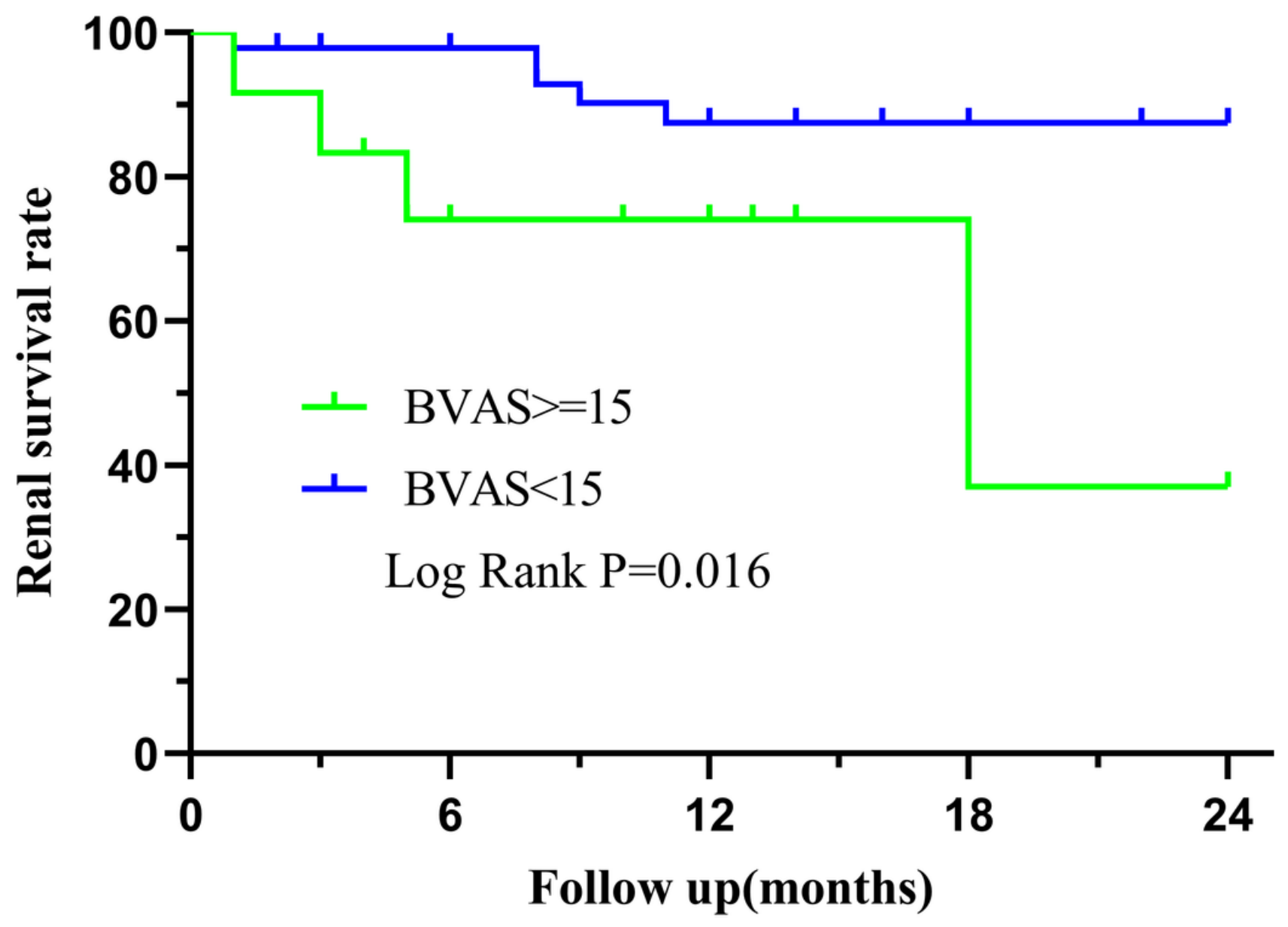

Figure 6

Comparison of the renal survival rate of patients with BVAS $\geq 15$ and patients with BVAS $<15$. 


\section{UACR}

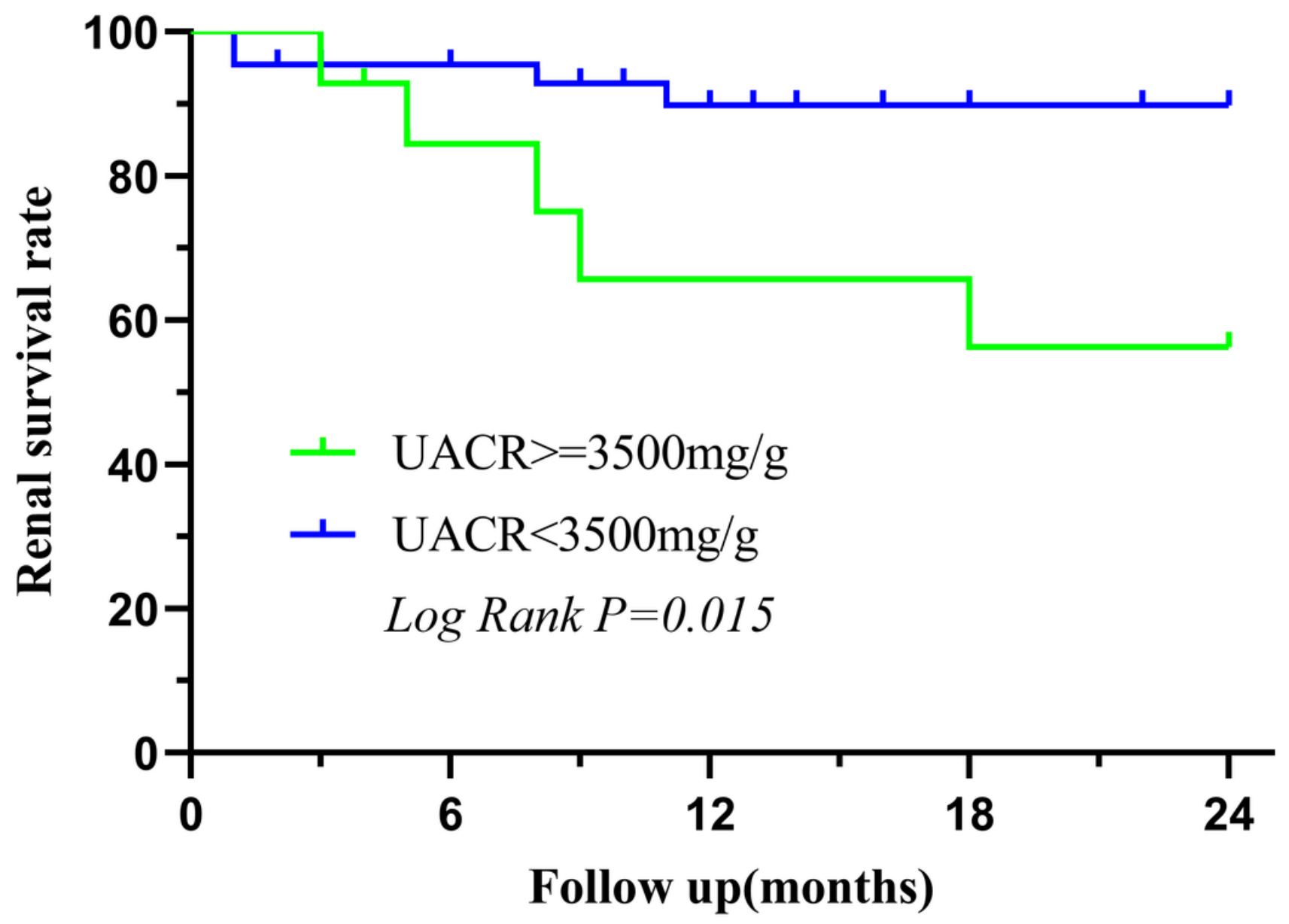

Figure 7

Comparison of the renal survival rate of patients with UACR $\leq 3500 \mathrm{mg} / \mathrm{g}$ and patients with UACR $>3500$ $\mathrm{mg} / \mathrm{g}$. 


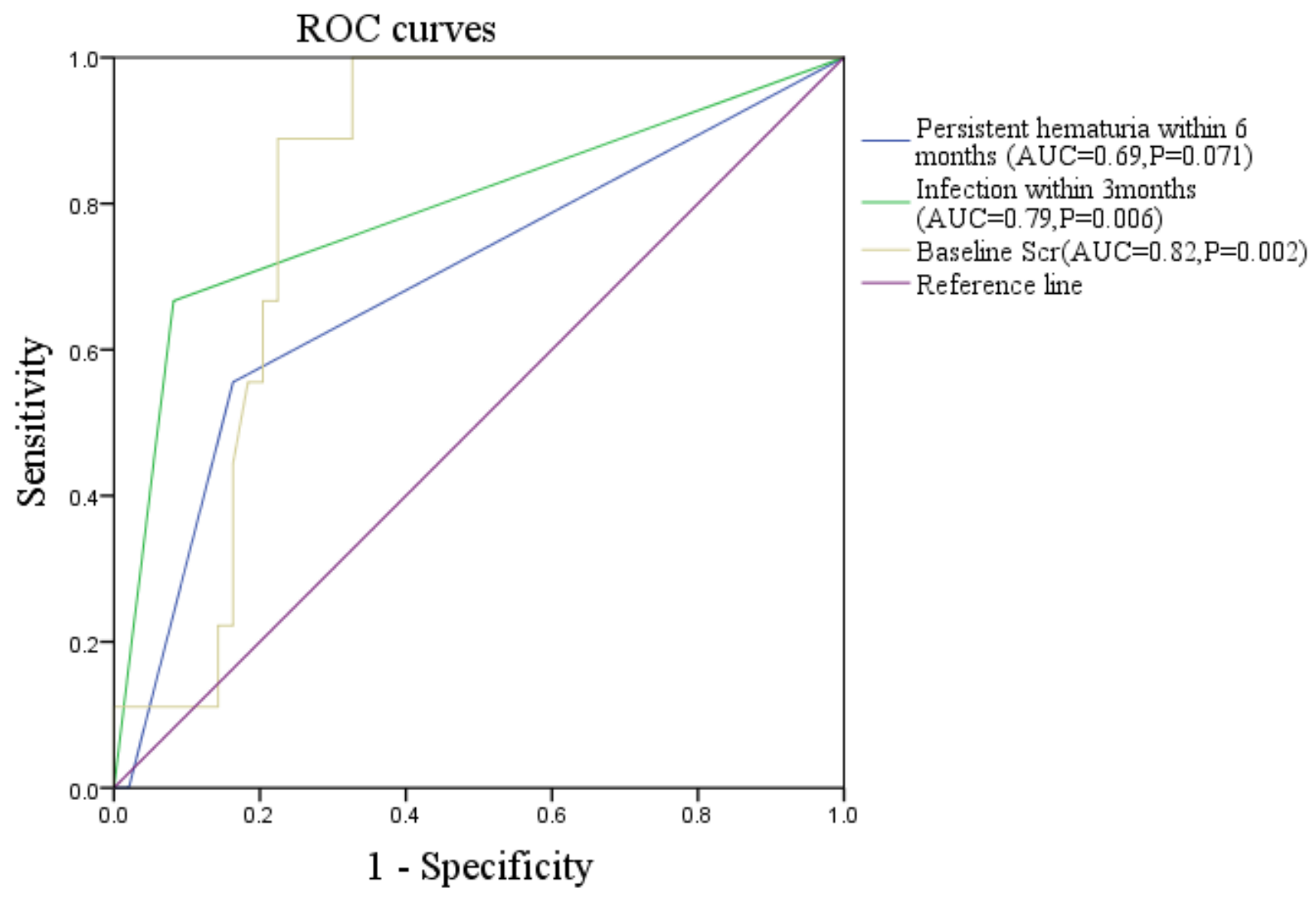

Figure 8

Evaluation of the predictive value of baseline creatinine, secondary infection within 3 months, and persistent hematuria within 6 months on ESRD 